American Journal of Pharmacology and Toxicology 6 (1): 1-4, 2011

ISSN 1557-4962

(C) 2011 Science Publications

\title{
Case Report of Severe Injection Site Reaction Associated with Intramuscular Naltrexone
}

\author{
Jill A. Covyeou, Katharine E. Atto, Chadwick W. Howard and Jessica R. Schultz \\ Pharmacy Practice, Ferris State University College of Pharmacy, \\ Family and Child Health Clinic, 1458 W. Center Rd, Suite 1, \\ Essexville, MI, USA 48732
}

\begin{abstract}
Problem statement: To report a case of severe injection site reaction at the site of an IM naltrexone injection in a 30 year old female. Approach: A 30 year old female, being treated with naltrexone extended-release injectable suspension for alcohol dependence, presented with a mass on her left upper gluteal area at the site of injection. The injectable naltrexone was discontinued, however the mass persisted. Upon referral to a general surgeon, it was determined that the mass was a hematoma or subcutaneous tissue necrosis secondary to naltrexone injection. Results: Naltrexone extended-release injection is Food and Drug Administration (FDA) approved for maintaining abstinence in alcohol dependency. It has been shown to increase the time to relapse compared to placebo, decrease the number of drinking days compared to placebo and decrease the number of heavy drinking days as compared to placebo. There have been no published case reports of severe injection site reaction associated with injectable naltrexone; however there has been a MedWatch alert warning of the risk of injection site reactions after receiving the injection. Conclusion: Patients receiving the injectable naltrexone should carefully monitor the injection site for signs of injection site reaction.
\end{abstract}

Key words: Injection site reaction, adverse drug reaction, intramuscular naltrexone

\section{INTRODUCTION}

Case report: A 30-year-old Caucasian female reported to the clinic with a chief complaint of a mass on her left upper gluteal area. The patient has a body mass index of $22.8 \mathrm{~kg} \mathrm{~m}^{-2}$ and has no known drug allergies. Significant past medical history includes an episode of acute alcohol intoxication two years prior (blood alcohol content of $0.34 \%$ ) and a history of mild depression with anxiety. Social history is significant for alcohol dependence, with many reported episodes of binging and referral to a behavioral health clinic for her alcohol dependence. At the time, she had abstained from alcohol over the past three months with the help of behavioral health counseling. She reported this gluteal mass (at the injection site) to the clinic approximately 45 days after receiving a naltrexone injection (Vivitrol ${ }^{\circledR}$ Alkermes Inc, Cambridge, MA) at the behavioral health clinic. Her medications at the time of the injection included escitalopram $10 \mathrm{mg}$ daily, hydroxyzine and an oral contraceptive.

The history of the gluteal mass began approximately one month after receiving a naltrexone injection. The patient began noticing signs of a painful, large welt at the injection site. She presented to the emergency room and was given cephalexin. After one week of treatment, the mass was unresponsive to cephalexin and still bothersome. She then returned to the emergency room and was given trimethoprim/sulfamethoxazole. A radiographic exam revealed edema and erythema, but no fluid collection to support diagnosis of an abscess. Edema was also noted in the left lower back. All other findings were normal.

At the follow up clinic visit, 10 days later, (approximately 45 days after the naltrexone injection), the patient stated no improvements with antibiotics and continued tenderness in the left upper gluteal area. At this time, the mass was noted to be solid, measuring approximately $7.6 \times 10.2 \mathrm{~cm}$ ( 3 inches by 4 inches) in size. It had not increased or decreased in size, despite the patient using ice for attempted relief. The site was mildly erythematous in nature, non-fluctuant and tender to palpitation. Range of motion, sensation and pulses in the lower extremities were intact. All other components of the physical exam were normal. No pertinent labs for this visit were obtained. Vital signs included a blood pressure of $128 / 90 \mathrm{~mm} \mathrm{Hg}$, pulse of 74 beats per minute, respirations of 16 breaths per minute, temperature of 97.8 degrees Fahrenheit and a pain scale score of 8 out of 10 . The visit concluded with a referral to a general surgeon for a second opinion, with possible surgical removal of the mass.

Corresponding Author: Jill A. Covyeou, Ferris State University College of Pharmacy, Family and Child Health Clinic, 1458 W. Center Rd, Suite 1, Essexville, MI 48732 
Am. J. Pharm. \& Toxicol., 5 (4): 1-4, 2011

Two weeks later, the patient presented for the surgical consultation. The mass was soft, edematous and erythematous and continued to be uncomfortable for the patient. The patient reported that the mass was firmer, but less uncomfortable. The physical exam revealed an $8 \times 9 \mathrm{~cm}$ firm lesion, which was fairly movable. The left lower extremity had good flexion and extension at the knee, normal sensation and good posterior tibial and dorsalis pedis pulses. The general surgeon's impression of the mass revealed a high possibility of the mass being necrotic scar tissue, secondary to the naltrexone injection. His plan was to either surgically excise the mass or continue to observe it. The patient expressed interest in having the mass excised. An MRI was ordered to ensure the absence of major muscles or significant nerves and to explore what tissue was specifically affected. If the mass involved only the subcutaneous tissue, the general surgeon would remove it. If the mass involved deeper tissues, an orthopedic surgeon would be consulted.

Three weeks later, (approximately three months after receiving the naltrexone injection), an MRI of the pelvis was performed with and without contrast. The MRI revealed a large heterogeneous appearing mass in the left gluteal area, measuring $5.5 \times 7 \mathrm{~cm}$. Some fluid and fatty contents were noted. Wall enhancements were involved, but no internal enhancements. The differential diagnosis was either a hematoma or subcutaneous tissue necrosis, both secondary to the naltrexone injection. Based on the MRI examination and patient's presentation, the radiologist reported that it was not a soft tissue tumor. On the Naranjo adverse drug reaction probability scale, it was also considered probable that the reaction was due to the naltrexone (Naranjo et al., 1991).

After the above visit, the patient's care was transferred to the general surgeon for surgical removal of the large mass.

\section{DISCUSSION}

Naltrexone is an opioid antagonist, FDA (Food and Drug Administration) approved for maintaining abstinence in alcohol dependency. The dosage of naltrexone for alcohol dependency is $380 \mathrm{mg}$ delivered Intramuscularly (IM) every 4 weeks (gluteal injection, alternating buttocks per injection) using the carton components provided (Alkermes, 2009). Patients should not be drinking alcohol at the time of therapy initiation. The patient presented in the above case report had been abstinent from alcohol for several months before receiving the injection.

Naltrexone is a competitive opioid antagonist with highest affinity for the mu receptor. The mechanism for reducing alcohol consumption is not entirely understood, however it may involve endogenous opioid pathways. When an alcohol-dependent person consumes alcohol, dopamine levels are elevated. This elevation is a result of $\beta$-endorphin release, which stimulates dopamine release directly in the nucleus accumbens and indirectly (in the ventral tegmental area) by inhibiting the activity of GammaAminobutyric Acid (GABA) neurons. Naltrexone reverses both of these actions in alcohol dependent patients to decrease the positive feedback of alcohol consumption (Johnson, 2007; Anton, 2008).

There are conflicting results related to the efficacy of injectable naltrexone for the treatment of alcohol dependence. Some studies show little to no benefit. For example, one trial found no significant differences between the treatment and placebo groups in terms of relapse rate, percentage of drinking days or number of drinks per drinking day (Krystal et al., 2001). Another trial concluded that injectable naltrexone did decrease the rate of heavy drinking by approximately $25 \%$, but had no effect on the rate of risky drinking or drinking days (Garbutt et al., 2005).

However, several studies did show a positive result. One of the first trials to evaluate the safety and efficacy of injectable naltrexone showed an increase in the mean percentage of days abstinent, a reduction in the number of drinks per drinking day and a reduction in the percentage of heavy drinking days (Johnson et al., 2004). Another trial found that injectable naltrexone increased time to first drinking day but did not have an effect on time to first heavy drinking day (Kranzler et $a l ., 2004)$. One trial found that only patients who were abstinent from alcohol before treatment showed a benefit (O'Malley et al., 2007). Furthermore, a recent trial demonstrated fewer drinks per day, fewer drinking days per month and decreased rates of heave drinking days (Lee et al., 2010) One of the largest trials evaluating the efficacy of injectable naltrexone was the COMBINE trial, which evaluated the effect of injectable naltrexone in combination with behavioral intervention, acamprosate, or both. The conclusion was that injectable naltrexone plus behavioral intervention had a positive effect on number of days abstinent and injectable naltrexone alone had a positive effect on the time to first heavy drinking day (Anton et al., 2006).

The most common adverse reactions reported in trials were injection site reactions. These included inflammation, pruritus, induration, pain and drainage (Krystal et al., 2001; Garbutt et al., 2005; Johnson et al., 2004; Kranzler et al., 2004; Comer et al., 2006; Galloway et al., 2005; Lee et al., 2010). Other side effects included gastrointestinal upset (such as nausea and abdominal pain) or nervous system problems (such as fatigue, headache and dizziness) (Krystal et al., 2001; 
Garbutt et al., 2005; Johnson et al., 2004; Kranzler et al., 2004; Comer et al., 2006; O'Malley et al., 1992; Lee et al., 2010).

Currently, there are no published reports of severe injection site reactions with injectable naltrexone. The FDA issued a MedWatch alert on 08/12/2008 describing the increased risk of injection site reactions relating to the use of injectable naltrexone (MedWatch, 2008. At the time of the alert, 196 cases of injection site reaction (of any kind) had been reported. Sixteen of these cases required surgical intervention (as in the case presented). The alert suggested that patients should be counseled to monitor their injection site and notify their physician if they notice any pain, swelling, tenderness, induration, bruising, pruritus, or redness at the injection site that does not improve or gets worse within two weeks of the injection. The alert also warns that, in cases of a worsening reaction, patients should be referred to a surgeon (which was the case with the patient presented).

Another sustained release naltrexone product, the naltrexone-poly9DL-lactide implant, also showed signs of local tissue reactions. In a study to assess the histologic changes associated with the naltrexone implants, researchers found that subjects developed localized tissue damage characterized by acute and chronic inflammation, tissue granulation, foreign body reaction and fibrosis (Hulse et al., 2005). While these reactions could be a result of other substances in the implant, animal research indicates that both naltrexone and the polymer are causative agents in the tissue's inflammatory response (Yamaguchi and Anderson, 1992).

As was stated previously, most trials evaluating the safety of injectable naltrexone reported injection site reaction to be the most common adverse effect. In one trial, all of the injection site reactions were rated as moderate in severity and resolved within 2-3 weeks. ${ }^{11}$ Another trial reported 27 injection site events in a study population of sixteen (Galloway et al., 2005). Most of these events were described as mild to moderate, with the most severe reaction resulting in drainage from the injection site. The package insert relates that 285 patients receiving injectable naltrexone in clinical trials reported at least one injection site reaction $(\mathrm{N}=440)$ while 106 patients receiving placebo reported at least one injection site reaction $(\mathrm{N}=214)$ (Alkermes and Vivitrol, 2009). Most reactions were mild but some cases were severe. In clinical trials, one patient developed an induration that enlarged after 4 weeks and eventually led to necrotic tissue requiring surgical excision (Alkermes, 2009) while another patient developed a painful, swollen, erythematous superficial and and subcutaneous lesion at the injection site that resolved with oral antibiotics and topical steroids.

Injectable naltrexone should be administered as an intra-muscular injection only. Both the package insert and the MedWatch alert state that inadvertent subcutaneous injection may increase the likelihood of injection site reactions. Women may also be at a higher risk for injection site reactions due to higher gluteal fat thickness (Alkermes, 2009; MedWatch, 2008). Therefore, professionals who administer this injection are advised to only use the custom needle provided in the carton to ensure that the medication is injected into the muscle and not into subcutaneous areas.

\section{CONCLUSION}

This is a case of a patient presenting with a severe injection site reaction due to naltrexone injectable suspension. The most common adverse effect associated with injectable naltrexone is injection site reaction, although severe reactions are rare. In this case the patient was a woman, putting her at higher risk of injection site reaction due to inadvertent injection into subcutaneous tissue. In any case, patients being treated with injectable naltrexone should be monitored closely for injection site reactions.

\section{REFERENCES}

Alkermes and Vivitrol, 2009. (Naltrexone for Extended-Releaseinjectable Suspension) Package Insert. Cambridge, MA.

Anton, R., 2008. Naltrexone for the management of alcohol dependence. New England J. Med., 359: 715-21.

Anton, R., S. O'Malley and D. Ciraulo, 2006. Combined pharmacotherapies and behavioral interventions for alcohol dependence: the combine study: A randomized controlled trial. JAMA, 295: 2003-17.

Comer, S., M. Sullivan and E. Yu, 2006. Injectable, sustained-release naltrexone for the treatment of opioid dependence. Arch. Gen. Psychiatry, 63: 210-8.

Galloway, G., M. Koch, R. Cello and D. Smith, 2005. Pharmacokinetics, safety and tolerability of a depot formulation of naltrexone in alcoholics: an openlabel trial. BMC Psychiatry, 5.

Garbutt, J., H. Kranzler and S. O'Malley, 2005. Efficacy and tolerability of long-acting injectable naltrexone for alcohol dependence: a randomized controlled trial. JAMA, 293: 1617-25. 
Hulse, G.K., V. Stalenberg and D. McCallum, 20005. Histological changes over time around the site of sustained release naltrexone-poly(DL-lactide) implants in humans. J. Cont. Release, 108: 45-55.

Johnson, B., 2007. Naltrexone long-acting formulation in the treatment of alcohol dependence. Ther. and Clin. Risk Manage., 3: 741-9.

Johnson, B., N. Ait-Daoud and H.J. Aubin, 2004. A pilot evaluation of the safety and tolerability of repeat dose administration of long-acting injectable naltrexone (vivitrex) in patients with alcohol dependence. Alcohol. Clin. Exp. Res., 28: 1356-61.

Kranzler, H., D. Wesson and L. Billot, 2004. Naltrexone depot for treatment of alcohol dependence: a multicenter, randomized, placebocontrolled clinical trial. Alcohol. Clin. Exp. Res., 28: 1051-59.

Krystal, J., J. Cramer, W. Krol, G. Kirk and R. Rosenheck et al., 2001. Naltrexone in the treatment of alcohol dependence. New England J. Med., 345: 1734-9.

Lee, J., E. Grossman, D. DiRocco, A. Truncali, K. Hanley, D. Stevens, J. Rotrosen, M. Gourevitch. 2010 Extended-release naltrexone for treatment of alcohol dependence in primary care. Journal of Substance Abuse Treatment, 39: 14-21.
MedWatch Naltrexone injection site reactions [naltrexone for extended-release injectable suspension (marketed as vivitrol)]- healthcare prefessional sheet text version. Food and Drug Administration

8/12/2008;http://www.fda.gov/ForConsumers/Cons umerUpdates/ucm126446.htm.

Naranjo, C.A., U. Busto and E.M. Sellers, 1981. A method for estimating the probability of adverse drug reactions. Clin. Pharmacol. Ther., 30: 239-45.

O'Malley, S., A. Jaffee, G. Chang, R. Schottenfeld and R. Maeyer et al., 1992. Naltrexone and coping skills therapy for alcohol dependence. Arch. Gen. Psychiatry, 49: 881-7.

O'Malley, S., J. Garbutt, D. Gastfriend, Q. Dong and H. Kranzler, 2007. Efficacy of extended-release naltrexone in alcohol-dependent patients who are abstinent before treatment. J. Clin. Psychopharmacol., 27: 507-12.

Yamaguchi, K. and J. Anderson, 1992. Biocompatibility studies of naltrexone sustained release formulations. J. Contr. Release, 19: 299-314. 